\title{
Inhomogeneous Big Bang Nucleosynthesis and Mutual Ion Diffusion
}

\author{
Elina Keihänent \\ Department of Physical Sciences, University of Helsinki, P.O.Box 64, FIN-00014 University of Helsinki, Finland
}

\begin{abstract}
We present a study of inhomogeneous big bang nucleosynthesis with emphasis on transport phenomena. We combine a hydrodynamic treatment to a nuclear reaction network and compute the light element abundances for a range of inhomogeneity parameters. We find that shortly after annihilation of electron-positron pairs, Thomson scattering on background photons prevents the diffusion of the remaining electrons. Protons and multiply charged ions then tend to diffuse into opposite directions so that no net charge is carried. Ions with $Z>1$ get enriched in the overdense regions, while protons diffuse out into regions of lower density. This leads to a second burst of nucleosynthesis in the overdense regions at $T<20 \mathrm{keV}$, leading to enhanched destruction of deuterium and lithium. We find a region in the parameter space at $2.1 \times 10^{-10}<\eta<5.2 \times 10^{-10}$ where constraints ${ }^{7} \mathrm{Li} / \mathrm{H}<10^{-9.7}$ and $\mathrm{D} / \mathrm{H}<10^{-4.4}$ are satisfied simultaneously.

PACS numbers: 26.35.+c, 98.80.Ft, 05.60.Cd
\end{abstract}

\section{INTRODUCTION}

Inhomogeneous big bang nucleosynthesis (IBBN) has been studied in several papers [1] 18 . In IBBN the baryon density is assumed to be inhomogeneous during nucleosynthesis. The inhomogeneity could be the result of a first-order phase transition occurring before BBN, or of some unknown physics possibly connected with inflation.

The effects on light element production depend strongly on the length scale of the inhomogeneity. It is well known that there is a so-called "optimal scale", at which the production of ${ }^{4} \mathrm{He}$ is reduced with respect to the homogeneous case, due to differential diffusion of protons and neutrons.

The first studies on IBBN concentrated on the reduced ${ }^{4} \mathrm{He}$ production and disregarded dissipative phenomena other than diffusion. Later works consider also other transport phenomena. The collective hydrodynamic expansion of the high-density regions was first addressed by Alcock et al. 19. Jedamzik and Fuller 20] give a detailed study of dissipative processes at temperatures ranging from $T \approx 100 \mathrm{GeV}$ to $T \approx 1 \mathrm{keV}$, including diffusion, hydrodynamic expansion, and photon inflation.

The mutual diffusion of isotopes, however, has to our knowledge not been properly accounted for previously. Diffusion of one ion species is not restricted by collisions with another species, if both are moving into same direction with same fluid velocity. On the other hand, momentum transfer is enhanched between two fluid components flowing into opposite directions.

Accurate treatment of transport phenomena has become increasingly important, since several estimations on the primordial ${ }^{7} \mathrm{Li}$ abundance indicate a low primordial ${ }^{7} \mathrm{Li} / \mathrm{H}$, which is difficult to accomodate in standard big bang nucleosynthesis (SBBN). Lithium is produced quite late in nucleosynthesis, and its yield is therefore particularly sensitive to the late-time transport phenomena such as ion diffusion and hydrodynamic expansion of the overdense regions.

In this work we study inhomogeneous big bang nucleosynthesis with emphasis on ion transport. We treat the primordial plasma as a fluid, and handle the dissipation of the baryon inhomogeneity through hydrodynamic equations. This allows us to take into account the effects of mutual diffusion. We discuss the hydrodynamics of the primordial plasma Section II. In Section III we present results from numerical simulations. In the last two sections we compare the predicted isotope yields with observations and give our conclusions.

Throughout this paper we use the natural unit system where $c=\hbar=k_{B}=1$.

\section{DISSIPATION OF BARYON INHOMOGENEITY}

\section{A. Ions}

Consider the evolution of a density fluctuation in the baryonic component of the primordial plasma. We are interested in the temperature range $T \sim 10 \mathrm{MeV}-1 \mathrm{keV}$. We treat each isotope as a separate fluid. We write down the hydrodynamic equations for isotope $i$ :

$$
\begin{aligned}
& \frac{\partial n_{i}}{\partial t}=-\nabla \cdot\left(n_{i} \mathbf{v}_{i}\right)+\left.\frac{\partial n_{i}}{\partial t}\right|_{\text {reac }} \\
& \frac{\partial \mathbf{q}_{i}}{\partial t}=-T \nabla n_{i}+\sum_{j \neq i} \mathbf{F}_{i j}+\mathbf{F}_{i e}-n_{i} Z_{i} e \mathbf{E}
\end{aligned}
$$

Here $n_{i}$ and $\mathbf{q}$ denote, respectively, the number and momentum density of isotope $i$. We have ignored secondorder terms in fluid velocity $\mathbf{v}_{i}$, which is assumed to be small. We use the non-relativistic formula for pressure $P=n T$ and assume that temperature is nearly homogeneous. As pointed out in [20], the fluctuations in temperature are of the order of the baryon-to-photon ratio $\sim 10^{-9}$. 
The last term in Eq. (1) represents production or destruction of ions via nuclear rections. Terms $\mathbf{F}_{i e}$ and $\mathbf{F}_{i j}$ represent momentum transfer due to collisions on electrons or other fluid components. The last term in Eq. (2) represents an electric field, which is present if there is a departure from local charge neutrality. In the following we evaluate explicit formulae for the collision terms $\mathbf{F}$.

\section{Scattering between non-relativistic particles.}

The momentum transfer between two non-relativistic fluid components close to thermal equilibrium is given by 21

$$
\begin{aligned}
\mathbf{F}_{k j}= & n_{k} n_{j} \iint \mathbf{d}^{\mathbf{3}} \mathbf{p}_{k} \mathbf{d}^{\mathbf{3}} \mathbf{p}_{j} \times \\
& f_{k}\left(\mathbf{p}_{k}\right) f_{j}\left(\mathbf{p}_{j}\right)\left|\mathbf{u}_{j}-\mathbf{u}_{k}\right| \sigma_{k j}^{\mathrm{t}}\left(p_{j k}\right) \mathbf{p}_{j k},
\end{aligned}
$$

where $f_{k}(\mathbf{p})$ is the momentum distribution of particle $k$, such that $n_{k} f_{k}(\mathbf{p})$ gives the phase space density, $\mathbf{u}_{j}-\mathbf{u}_{k}$ is the relative velocity, $\mathbf{p}_{j k}$ is the center-of-mass momentum, and

$$
\sigma_{k j}^{\mathrm{t}}=\int \frac{d \sigma_{k j}}{d \Omega}(1-\cos (\theta)) d \Omega
$$

is the transport cross section. Assuming a small deviation from the Maxwellian distribution $f_{k}^{0}$,

$$
f_{k}(\mathbf{p})=f_{k}^{0}(\mathbf{p})\left(1+\frac{\mathbf{v}_{k} \cdot \mathbf{p}}{T}\right)
$$

where $\mathbf{v}_{k}=\left\langle u_{k}\right\rangle$ is the fluid velocity, we obtain

$$
\mathbf{F}_{k j}=-n_{k} n_{j} S_{k j}\left(\mathbf{v}_{k}-\mathbf{v}_{j}\right)
$$

where

$$
S_{k j}=\frac{8}{3}\left(\frac{2 T \mu}{\pi}\right)^{1 / 2} \tilde{\sigma}_{k j}(T)
$$

Here $\mu$ is the reduced mass and the thermally averaged cross section is given by

$$
\tilde{\sigma}_{k j}(T)=\frac{1}{(2 \mu T)^{3}} \int_{0}^{\infty} \exp \left(-\frac{k^{2}}{2 \mu T}\right) k^{5} \sigma_{k j}^{\mathrm{t}}(k) d k,
$$

where $k$ is the center-of-mass momentum.

Let us apply the above results to neutron-proton and ion-ion scattering. At low energies (below a few MeV) the neutron-proton interaction is dominated by s-wave scattering. The cross section is given by 22

$$
\sigma_{n p}(k)=\frac{\pi a_{s}^{2}}{\left(a_{s} k\right)^{2}+\left(1-\frac{1}{2} r_{s} a_{s} k^{2}\right)^{2}}+\frac{3 \pi a_{t}^{2}}{\left(a_{t} k\right)^{2}+\left(1-\frac{1}{2} r_{t} a_{t} k^{2}\right)^{2}}
$$

with $a_{s}=-23.71 \mathrm{fm}, a_{t}=5.432 \mathrm{fm}, r_{s}=2.73 \mathrm{fm}$, and $r_{t}=1.749 \mathrm{fm}$. At zero-energy limit the cross section approaches the value 20440 mbarn. The thermally averaged cross section (\&) can be evaluated numerically.
The transport cross section for Coulomb scattering between nonrelativistic ions is given by

$$
\sigma^{\mathrm{t}}=4 \pi\left(Z_{i} Z_{j} \alpha\right)^{2} \frac{\mu^{2}}{k^{4}} \Lambda
$$

where $\Lambda$ is the Coulomb logarithm [23,24. The thermal cross section (8) becomes $\tilde{\sigma}=\pi\left(Z_{i} Z_{j} \alpha\right)^{2} \Lambda /\left(2 T^{2}\right)$. We then have

$$
S_{\mathrm{ij}}=\frac{4 \pi}{3}\left(\frac{2 \mu_{i j} T}{\pi}\right)^{1 / 2} \frac{\left(Z_{i} Z_{j} \alpha\right)^{2} \Lambda}{T^{2}} .
$$

\section{Scattering on electrons.}

The collisional force excerted on a heavy particle $k$ moving with velocity $\mathbf{v}_{k}$ through a thermal background of light particles $j$ is given by

$$
\mathbf{F}_{k j}=n_{k} \int \mathbf{d}^{3} \mathbf{p} \rho_{j}(\mathbf{p}) \frac{|\mathbf{p}|}{E} \sigma_{k j}^{\mathrm{t}}(\mathbf{p}) \mathbf{p}=-\frac{1}{b_{k j}} n_{k} \mathbf{v}_{k} .
$$

This equation relates the force to the mobility $b_{k j}$ [23,25]. Here $\rho_{j}(\mathbf{p})$ is the phase space density of particle $j$ in the frame of particle $k$. Assuming a thermal distribution $\rho_{j}^{0}$ for particle $j$ in laboratory frame, we have $\rho_{j}(\mathbf{p})=$ $\rho_{j}^{0}(E+\mathbf{p} \cdot \mathbf{v})$, and

$$
-b_{n e}^{-1}=\int \mathbf{d}^{\mathbf{3}} \mathbf{p} \frac{d \rho_{j}^{0}(E)}{d E} \frac{p^{3}}{3 E} \sigma_{k j}^{\mathrm{t}}(\mathbf{p}) .
$$

Neutrons interact with electrons through their magnetic moment. The transport cross section is [2]

$$
\sigma_{n e}^{\mathrm{t}}=3 \pi \frac{\alpha^{2} \kappa^{2}}{m_{n}^{2}}=8.07 \times 10^{-4} \text { mbarn. }
$$

where $\kappa=-1.91$ is the anomalous magnetic moment of the neutron. Using MB statistics for electrons we obtain for the mobility

$$
b_{n e}^{-1}=\frac{8}{3}\left(\frac{2 m_{e} T}{\pi}\right)^{1 / 2} \frac{K_{2.5}(z)}{K_{2}(z)} \sigma_{n e}^{\mathrm{t}} n_{e} .
$$

where $K$ are modified bessel functions.

The differential cross section for a relativistic electron scattering on an ion with charge $Z$ is given by the Mott formula 26]

$$
\frac{d \sigma}{d \Omega}=\frac{(Z \alpha)^{2} E_{e}^{2}}{4 k^{4} \sin ^{4}(\theta / 2)}\left(1-\beta^{2} \sin ^{2}(\theta / 2)\right) .
$$

where the factor $1-\beta^{2} \sin ^{2}(\theta / 2)$ is the relativistic spin correction. This gives the transport cross-section

$$
\sigma_{i e}^{\mathrm{t}}(k)=4 \pi(Z \alpha)^{2} \frac{m_{e}^{2}+k^{2}}{k^{4}} \Lambda .
$$

where $\Lambda$ is the Coulomb logarithm. Using again MB statistics we obtain

$$
b_{i e}^{-1}=\frac{4 \pi T}{3} \frac{\left(z^{2}+2 z+2\right)}{K_{2}(z) e^{z}} \frac{\left(Z_{i} \alpha\right)^{2} \Lambda n_{e}}{m_{e}^{2}} .
$$




\section{B. Electrons}

Thermal electron-positron pairs annihilate at temperatures $T \approx 1 \mathrm{MeV}-20 \mathrm{keV}$. The remaining electrons must be treated as one fluid component.

For non-relativistic electrons we have

$$
\begin{aligned}
\frac{\partial n_{e}}{\partial t} & =-\nabla \cdot\left(n_{e} \mathbf{v}_{e}\right) \\
\frac{\partial \mathbf{q}_{e}}{\partial t} & =-T \nabla n_{e}+\mathbf{F}_{e \gamma}+n_{e} e \mathbf{E} .
\end{aligned}
$$

The term $\mathbf{F}_{e \gamma}$ represents Thomson scattering on background photons 27,

$$
\mathbf{F}_{e \gamma}=-b_{e \gamma}^{-1} n_{e} \mathbf{v}_{e}=-\frac{4}{3} \sigma_{T} \epsilon_{\gamma} n_{e} \mathbf{v}_{e}
$$

where $\sigma_{T}=665$ mbarn is the Thomson cross section and $\epsilon_{\gamma}$ is the energy density of background photons.

Note that formula (21) is exactly valid only well after electron-positron annihilation, when photon mean free path is large compared with the inhomogeneity scale. Around $T \approx 20 \mathrm{keV}$ photons are still connected to the plasma. For precise treatment of this transition period, photons should be included as one fluid component.

Ions diffusing out from the high-density regions leave behing a negative net charge. That gives rise to an electric field, which forces electrons to move so as to restore the electrical neutrality 28]. Electrons are thus dragged along with ions. The motion of ions is restricted by the Thomson drag force (21), which acts on them indirectly through the electric field.

If we assume spherical symmetry, the electric field at a given location is determined by the total charge contained in the spherical region closer to the symmetry center. The rate of change of the field is then determined by the flux of charge through the sphere,

$$
\frac{\partial(e \mathbf{E})}{\partial t}=-4 \pi \alpha\left(n_{e} \mathbf{v}_{e}-\sum_{i} n_{i} Z_{i} \mathbf{v}_{i}\right) .
$$

The five differential equations (11), (2), (19), (20), and (22), together with the formulae for momemtum transfer terms, form the basis of our hydrodynamic simulations.

\section{Diffusion approximation}

It is interesting to look at how our hydrodynamic treatment relates to the common diffusion approximation, where the evolution of inhomogeneity is presented by a differential equation of the form

$$
\frac{\partial n}{\partial t}=\nabla \cdot(D \nabla n)
$$

Consider the steady-state solution of Eq. (2) in absence of an electric field,

$$
-T \nabla n_{i}-\sum_{j \neq i} n_{i} n_{j} S_{i j}\left(\mathbf{v}_{i}-\mathbf{v}_{j}\right)-b_{i e}^{-1} n_{i} \mathbf{v}_{i}=0
$$

If we ignore the motion of particle species other than $i$ $\left(v_{j} \approx 0\right.$ for $\left.j \neq i\right)$, equation (24) and the continuity equation (11) together lead to a diffusion equation of the form (23), with diffusion constants given by $D_{i e}=b_{i e} T$ for scattering on electrons, and $D_{i j}=T /\left(n_{j} S_{i j}\right)$ for scattering between nuclei.

We note here that the neutron-proton and neutronelectron diffusion constants calculated this way coincide with those given in 18. Also the proton-electron constant is in agreement at the limit $\Lambda \gg 1$.

The diffusion equation describes well the motion of a fluid if the background fluid is stationary, so that its mutual motion can be ignored. We refer to this as the approximation of independent diffusion. This approximation is valid in the case of diffusion of neutrons, which are much more mobile than the ions and electrons they scatter on. The diffusion equation also describes well the motion of ions at high temperatures $(T \gg 20 \mathrm{keV})$, where the dominant scattering process for ions is Coulomb scattering on background electrons. Due to their large number the electrons can be regarded as a stationary background. At lower temperatures the situation is more complicated. Ions gain or lose momentum in collisions on other ion components, which move with comparable fluid velocities. Thus the mutual motion of the fluid components cannot be neglected. The situation is further complicated by electron drag: electrons are dragged along with ions so that charge neutrality is maintained.

\section{SIMULATIONS}

We have written an inhomogeneous nucleosynthesis code where a nuclear reaction network is coupled to hydrodynamic equations. We assume spherical symmetry and use a non-uniform radial grid of 64 cells. The grid is adjusted according to the density profile so that the cells are smallest where the gradient of the baryon density is largest. We assume a simple initial geometry with a step-like density profile. The inner part of the simulation volume has a high baryon density $\eta_{h}$, and the outer part a low density $\eta_{l}$. The initial conditions are determined by four parameters: the volume fraction $f_{v}$ of the highdensity region, density contrast $R=\eta_{h} / \eta_{l}$, the average density $\eta=f_{v} \cdot \eta_{h}+\left(1-f_{v}\right) \cdot \eta_{l}$, and the radius of the simulation volume $r$. We give $r$ in comoving units in meters at $T=1 \mathrm{keV}$ temperature. One meter at $T=1 \mathrm{keV}$ corresponds to $4.26 \times 10^{6} \mathrm{~m}$ today. The baryon density is given as the baryon-to-photon ratio $\eta$, which is related to $\Omega_{b}$ through $\eta_{10}=10^{10} \eta=274 \Omega_{b} h^{2}$.

The code evolves 21 variables in each grid zone: the concentration and momentum density of $e, n, p, \mathrm{D},{ }^{3} \mathrm{H}$, ${ }^{3} \mathrm{He},{ }^{4} \mathrm{He},{ }^{6} \mathrm{Li},{ }^{7} \mathrm{Li}$, and ${ }^{7} \mathrm{Be}$, and the electric field. These variables are evolved by solving a set of $21 \times 64$ stiff differential equations in time steps proportional to the age 


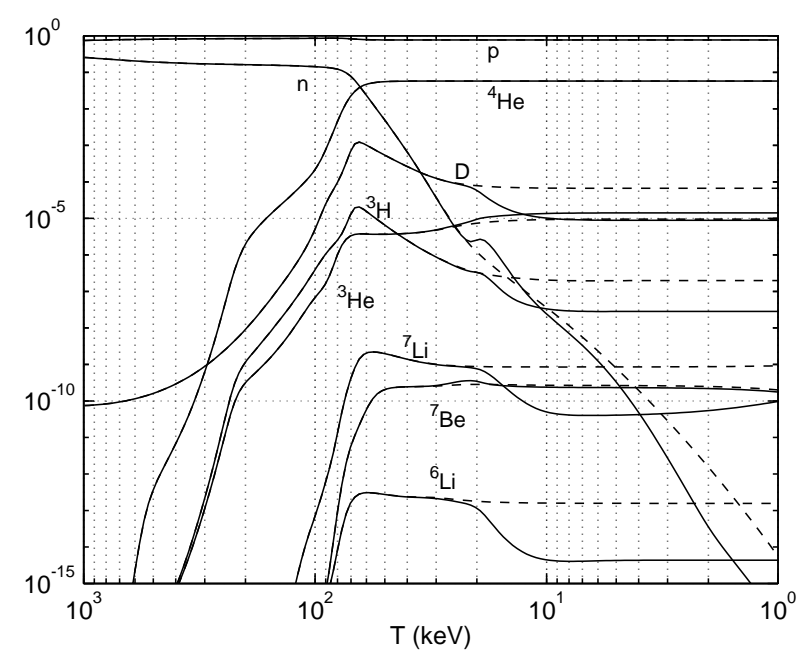

FIG. 1. Evolution of the abundances of light isotopes as a function of temperature, for simulation parameters $r=10^{7}$ $\mathrm{m}$ (at $1 \mathrm{keV}), \eta_{10}=6, f_{v}=0.01$, and $R=10^{6}$. The solid lines show results from a complete simulation. The dashed lines represent a simulation where the approximation of independent diffusion was applied. Separation of elements leads to a second burst of nucleosynthesis below $T<20 \mathrm{keV}$.

of the universe. This involves the solution of a band diagonal linear system of rank 1344 at every time step. The nuclear reaction rates include those given in the NACRE compilation [29]. The simulation is started at $T=10$ $\mathrm{MeV}$ and ends at $T=1 \mathrm{keV}$, or when all nuclear reactions have ceased. The final output consist of the average concentrations of $p, \mathrm{D},{ }^{3} \mathrm{He}$ (including $\left.{ }^{3} \mathrm{H}\right),{ }^{4} \mathrm{He},{ }^{6} \mathrm{Li}$, and ${ }^{7} \mathrm{Li}$ (including ${ }^{7} \mathrm{Be}$ ).

For comparison we also made a set of simulations where we mimicked the approximation of independent diffusion. We removed from the matrix all elements corresponding to mutual diffusion, i.e, terms that represent dependence of $\partial \mathbf{q}_{i} / \partial t$ on $\mathbf{v}_{j \neq i}$. We also forced a steady-state solution. The electron drag was taken into account by adding to the momentum loss of an ion with charge $Z_{i}$ the Thomson force that would act on $Z_{i}$ electrons moving with the same velocity. This approach can be written as

$$
\begin{aligned}
& \frac{\partial n_{i}}{\partial t}=-\nabla \cdot\left(n_{i} \mathbf{v}_{i}\right)+\left.\frac{\partial n_{i}}{\partial t}\right|_{\text {reac }} \\
& -T \nabla n_{i}-\sum_{j \neq i} n_{i} n_{j} S_{i j} \mathbf{v}_{i}-b_{i e}^{-1} n_{i} \mathbf{v}_{i}-b_{e \gamma}^{-1} Z_{i} n_{i} \mathbf{v}_{i}=0 .
\end{aligned}
$$

\section{A. Separation of elements}

Figure 11 shows the light element abundances plotted against temperature, for simulation parameters $r=10^{7}$ $\mathrm{m}, \eta_{10}=6$, and $f_{v}=0.01$. We compare results from a complete simulation, and from a simulation where we used the approximation (26). The complete simulation

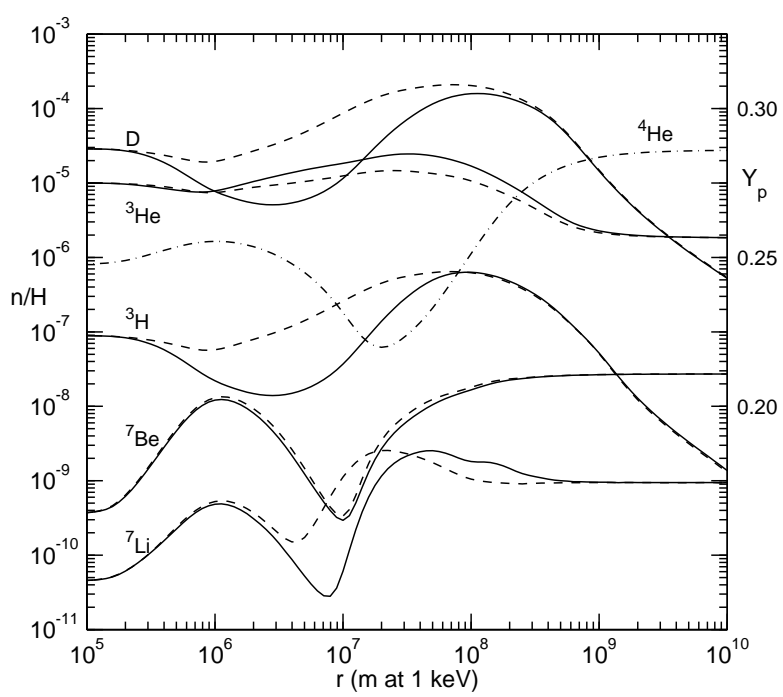

FIG. 2. Light element abundances as a function of $r$, for simulation parameters $\eta_{10}=6, f_{v}=0.01$, and $R=10^{6}$. The solid lines show results from a complete simulation. The dashed lines represent a simulation where mutual diffusion was ignored. The helium mass fraction $Y_{p}$ (dash-dotted line) is shown on linear scale (right y-axis). Other isotopes are shown on logarithmic scale (left y-axis) as ratio of number density to that of hydrogen.

shows a second burst of nucleosynthesis at $T \approx 20-10$ $\mathrm{keV}$, leading to destruction of ${ }^{7} \mathrm{Li}, \mathrm{D}$, and ${ }^{3} \mathrm{H}$.

This can be understood as follows. Diffusion of electrons is inefficient at temperatures $T \gg 1 \mathrm{keV}$ due to the frequent Thomson scattering on background photons. Thomson drag then resists the diffusion of ions which must drag electrons with them to maintain electrical neutrality. However, if we divide the motion of ions into two components, one that obeys the condition $\sum_{i} n_{i} \mathbf{v}_{i} Z_{i}=0$ and thus carries no charge, and one that does carry charge, only the latter is resisted by Thomson drag. Protons and helium ions, for instance, are allowed to diffuse into opposite directions in such a way that the total charge flux vanishes. This leads to a separation of elements. Heavier elements tend to get enriched in the high-density regions, while protons diffuse out. The nucleosynthesis process in the high-density region is enhanced by the increased concentration of heavier nuclei. This effect is responsible for the modified nucleosynthesis yields that our simulations show.

In Fig. 2 we show the isotope yields as a function of inhomogeneity length scale, for $f_{v}=0.01$ and $\eta_{10}=6$. Again, we show results both for a complete simulation (solid lines) and for a simulation with same parameters but with the approximation of independent diffusion. The complete simulation shows a clear decrease in the abundances of $\mathrm{D},{ }^{3} \mathrm{H}$, and ${ }^{7} \mathrm{Li}$, as compared to the diffusion approximation. Also the yields of ${ }^{3} \mathrm{He}$ and ${ }^{7} \mathrm{Be}$ are reduced, but not as clearly. The yield of ${ }^{4} \mathrm{He}$ is hardly affected. 
Lithium is destroyed via reaction ${ }^{7} \operatorname{Li}(p, a){ }^{4} \mathrm{He}$. The mean destruction channel for ${ }^{7} \mathrm{Be}$, instead, is via reaction ${ }^{7} \mathrm{Be}(n, p){ }^{7} \mathrm{Li}$. As this reaction requires free neutrons, which are not available after the main phase of nucleosynthesis, ${ }^{7} \mathrm{Be}$ is affected little in the second nucleosynthesis phase. The same holds for ${ }^{3} \mathrm{He}$, whose main reaction channel is ${ }^{3} \mathrm{He}(n, p){ }^{3} \mathrm{H}$.

The reduction in $\mathrm{D},{ }^{3} \mathrm{H}$ and ${ }^{7} \mathrm{Li}$ due to element separation is most efficient at scales somewhat smaller than the "optimal scale" at which the ${ }^{4} \mathrm{He}$ yield is minimized. The maximal ${ }^{4} \mathrm{He}$ reduction occurs at a scale at which neutrons diffuse maximally out from the high-density regions, but the later back-diffusion is not too efficient. At a somewhat smaller scale, back-diffusion of neutrons leads to synthesis of nuclei in a narrow zone surrounding the high-density region. There are then plenty of nuclei to be transported deeper into the high-density region, once the separation of elements begins at $T \sim 20 \mathrm{keV}$.

Some analytic considerations may be in place here. Consider the steady-state solution of Eqs. (2) and (20),

$$
\begin{aligned}
& -T \nabla n_{i}-\sum_{j} n_{i} n_{j} S_{i j}\left(\mathbf{v}_{i}-\mathbf{v}_{j}\right)-n_{i} Z_{i} e \mathbf{E}=0 \\
& -T \nabla n_{e}-b_{e \gamma}^{-1} n_{e} \mathbf{v}_{e}+n_{e} e \mathbf{E}=0 .
\end{aligned}
$$

We are interested in the temperature regime $T<20$ $\mathrm{keV}$ and have ignored terms that represent scattering on electrons. A measure of the distance over which $n_{e}$ can deviate from $\sum_{i} n_{i} Z_{i}$ is given by the Debye shielding distance $h=\left(4 \pi \alpha n_{e} / T\right)^{-1 / 2}$ [28], which is orders of magnitude smaller than the inhomogenity length scale. We can therefore assume that electrical neutrality holds at the scale of the inhomogeneity $\left(n_{e} \approx \sum_{i} n_{i} Z_{i}\right)$, and based on that eliminate the field $\mathbf{E}$.

The evolution of the inhomogeneity is particularly simple in two limiting cases. If the interaction between ions is strong compared with the electron-photon interaction $\left(n_{i} S_{i j} \gg b_{e \gamma}^{-1}\right)$, as is the case at late times $(T \ll 1 \mathrm{keV})$, the plasma behaves as a single fluid, moving with a collective velocity $\mathbf{v}_{e}$. Taking the sum of Eqs. (27) and (28) and using the electrical neutrality we find

$$
-T\left(\nabla n_{B}+\nabla n_{e}\right)-b_{e \gamma}^{-1} n_{e} \mathbf{v}_{e}=0 .
$$

This represents collective hydrodynamic motion of the plasma against Thomson drag [19,20]. With the approximation $n_{b} \approx n_{e}$ it leads to the diffusion equation for the baryon density, with diffusion constant $D_{h y d}=2 b_{e \gamma} T$.

In the opposite limiting case, when electron-photon scattering dominates over ion-ion scattering, the motion of electrons is suppressed by the Thomson drag. Ions then move under the condition that the net current carried by ions vanishes, $\sum_{i} n_{i} Z_{i} \mathbf{v}_{i}=0$. Consider for simplicity a system of two ion species only, say hydrogen and ${ }^{4} \mathrm{He}$. The steady-state solution now simplifies into

$$
T\left(Z_{1} \frac{\nabla n_{2}}{n_{2}}-Z_{2} \frac{\nabla n_{1}}{n_{1}}\right)=\left(\mathbf{v}_{1}-\mathbf{v}_{2}\right) S_{12}\left(n_{1} Z_{1}+n_{2} Z_{2}\right) .
$$
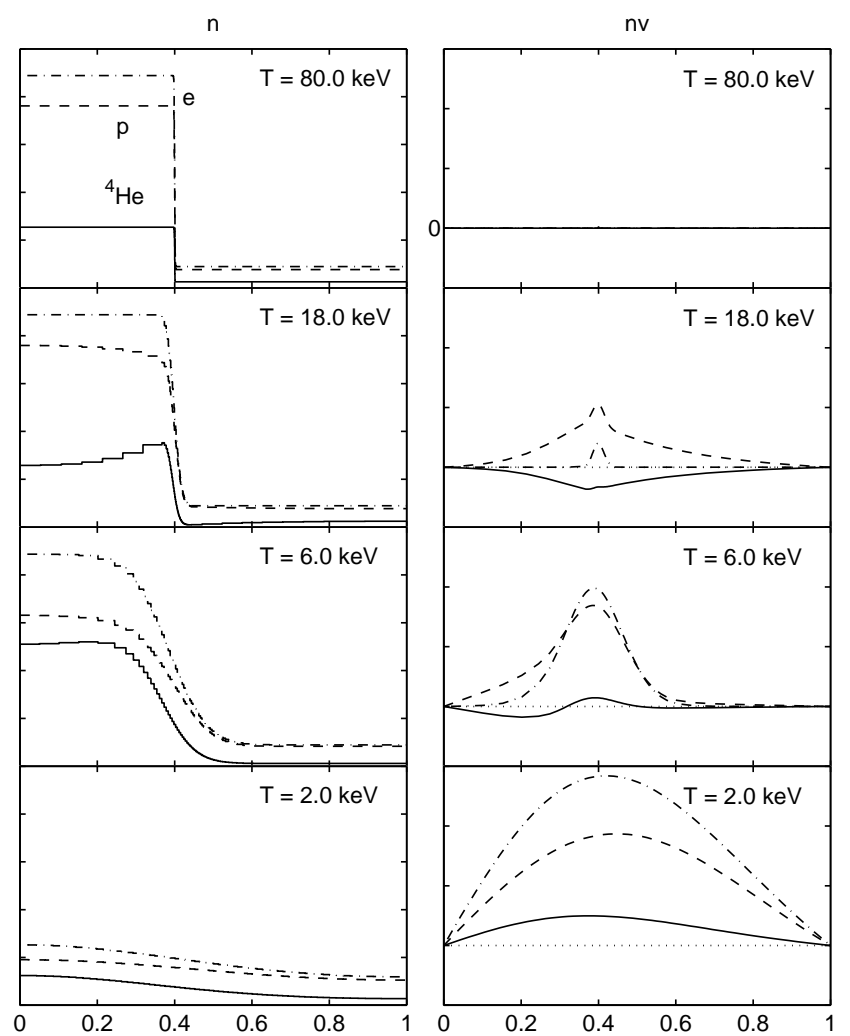

FIG. 3. Separation of elements. Number density (left) and $n \mathbf{v}$ (right) are shown for a run with with only protons and ${ }^{4}$ He present. Nuclear reaction were turned off. The simulation started with an initial profile with $R=10, r=10^{7} \mathrm{~m}$, $f_{v}=0.4^{3}$, and uniform helium mass fraction $Y_{p}=0.25$ (uppermost frame). We show the density profile and flux of ${ }^{4} \mathrm{He}$ (solid line), $p$ (dashed line), and $e$ (dash-dotted line) along the radial axis of the sperical simulation volume. Helium begins to concentrate in the high-density region at $T<20 \mathrm{keV}$. The concentration of ${ }^{4} \mathrm{He}$ in the center reaches a maximum around $T \approx 6 \mathrm{keV}$. The inhomogeneity is finally erased by collective hydrodynamic expansion against Thomson drag (last frame).

It is now easy to see that if two isotopes have the same initial inhomogeneity $\left(\nabla n_{1} / n_{1}=\nabla n_{2} / n_{2}\right)$, then the one with smaller charge will flow into the direction of negative density gradient, while the one with larger charge will move into the opposite direction. The isotope with larger charge will get concentrated into the high-density region.

In order to illustrate the separation of elements, we made a run with only protons and ${ }^{4} \mathrm{He}$ present. We started with a step-like initial profile with uniform helium mass fraction $Y_{p}=0.25$. The concentration profiles of the two elements and electrons, as well as density times velocity, at various temperatures are shown in Fig. 3 . Helium begins to concentrate in the high-density region at $T<20 \mathrm{keV}$. At $T=6 \mathrm{keV}$ the initial high-density region contains $62 \%$ of all helium nuclei, while in the beginning it contained $41 \%$. The inhomogeneity is erased when Thomson scattering becomes inefficient in restricting the collective motion of the plasma. 


\section{IBBN COMPUTATIONS AND CONFRONTATION WITH OBSERVATIONS}

The best way to evaluate the primordial ${ }^{7} \mathrm{Li}$ has for a long time been the so-called Spite plateau [30] observed in halo stars. There is still debate on how much the ${ }^{7} \mathrm{Li}$ in Spite plateau stars has depleted from the primordial abundance, and consequently, on the primordial ${ }^{7} \mathrm{Li}$ abundance. While some authors obtain an relatively high upper limit ${ }^{7} \mathrm{Li} / \mathrm{H}<4 \times 10^{-10} 31$, a number authors argue for a lower value 32 34. Ryan et al. 33] derive the range $-10.04<\log _{10}\left({ }^{7} \mathrm{Li} / \mathrm{H}\right)<-9.72$ for the primordial abundance. In SBBN this corresponds to $\eta_{10}=10^{10} \eta<4.2$. Suzuki et al. obtain an even tighter range $-9.97<\log _{10}\left({ }^{7} \mathrm{Li} / \mathrm{H}\right)<-9.77$. A recent study [35] gives an intermediate estimate $\log _{10}\left({ }^{7} \mathrm{Li} / \mathrm{H}\right)=$ $-9.76 \pm 0.056 \pm 0.06$.

The tight lithium limits of Ryan et al. and and Suzuki et al. are in conflict with the low deuterium estimations from QSO absorption systems [36 38]. O'Meara et al. obtain the range $\mathrm{D} / \mathrm{H}=(3.0 \pm 0.4) \times 10^{-5}$ from a combined study of four such systems. In SBBN this corresponds to $5.4<\eta_{10}<6.4$. The tight lithium limits are also in conflict with the recent Boomerang result $\eta \sim 6 \times 10^{-10} 39$.

In light of the above, it is interesting to note that the separation of elements due to Thomson drag leads to simultaneous destruction of ${ }^{7} \mathrm{Li}$ and $\mathrm{D}$.

We have computed the light element abundances for a range of inhomogeneity parameters. Figure 1 shows the yields of light isotopes as a function of length scale $r$ and volume fraction of the high-density region $f_{v}$, for $\eta=$ $6 \times 10^{-10}$. At small scales the results converge towards SBBN results. The smallest ${ }^{7} \mathrm{Li}$ yield ${ }^{7} \mathrm{Li} / \mathrm{H}=10^{-9.55}$ was obtained at $f_{v} \approx 10^{-1.5} \approx 0.032$ and $r \approx 7.1 \mathrm{~m}$. The SBBN value is ${ }^{7} \mathrm{Li} / \mathrm{H}=10^{-9.39}$.

In Fig. (5) we show the isotope yields as a function of $r$ and $\eta$, for $f_{v}=10^{-1.5}$ and $R=10^{6}$. The reduction in ${ }^{7} \mathrm{Li} / \mathrm{H}$ is more prominent at low $\eta$, due to the fact that at low $\eta$ most of the lithium is produced directly as ${ }^{7} \mathrm{Li}$, which is sensitive to the separation of elements. At high $\eta$ most of the lithium comes from ${ }^{7} \mathrm{Be}$, which is not affected as much.

In Fig. (6) we compare our results to a set of observational constraints on light element abundances. The simulation parameters are the same as in Figs. (5). For ${ }^{4} \mathrm{He}$ we choose conservative limits $0.23<Y_{p}<0.25$. For deuterium we select constraints $10^{-4.8}<\mathrm{D} / \mathrm{H}<10^{-4.4}$. The lower limit here comes from the present $\mathrm{D} / \mathrm{H}$ abundance in interstellar medium [40]. The upper limit is based on the two-sigma upper limit of the O'Meara estimation [36]. For lithium we choose a low limit ${ }^{7} \mathrm{Li} / \mathrm{H}<10^{-9.7}$. We also include the constraint ${ }^{3} \mathrm{He} / \mathrm{D}<1$. The lithium and deuterium constraints we have chosen are in conflict in SBBN. The upper limit to deuterium implies $\eta_{10}>4.8$ while the upper limit to ${ }^{7} \mathrm{Li}$ implies $\eta_{10}<4.2$. In IBBN we find a region in the parameter space, where all con-

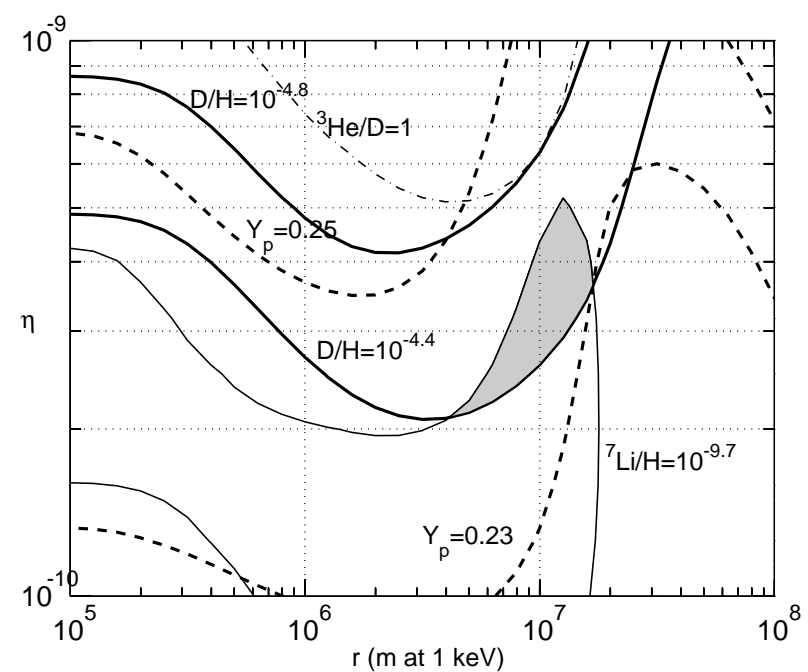

FIG. 6. Observational constraints on $(r, \eta)$ plane. The shaded region satisfies the constraints $0.23<Y_{p}<0.25$ (thick dashed lines), $10^{-4.8}<\mathrm{D} / \mathrm{H}<10^{-4.4}$ (thick solid lines), ${ }^{7} \mathrm{Li} / \mathrm{H}<10^{-9.7}$ (thin solid line), and ${ }^{3} \mathrm{He} / \mathrm{D}<1$ (dash-dotted line).

straints are satisfied simultaneously. The allowed region falls in the range $2.1<\eta_{10}<5.2$, corresponding to $0.008<\Omega_{b} h^{2}<0.019$. We note that if we apply the approximation of independent diffusion, the allowed region disappears.

\section{CONCLUSIONS}

We have studied inhomogeneous big bang nucleosynthesis with emphasis on transport phenomena. We combined a hydrodynamic treatment to a nucleosynthesis simulation. We found an effect that to our knowledge has been overlooked before: separation of elements due to Thomson drag. Thomson drag prevents the diffusion of the electron fluid shortly after electron-positron annihilation. Hydrogen and multiply charged elements then diffuse into opposite directions so that no net charge is carried. Helium and lithium get concentrated into highdensity regions, which leads to enhanched nucleosynthesis and destruction of ${ }^{7} \mathrm{Li}, \mathrm{D}$, and ${ }^{3} \mathrm{He}$. The effect is important at length scales from $10^{5}$ to $10^{9}$ meters at 1 $\mathrm{keV}$ temperature, corresponding to $10^{-5}-0.1 \mathrm{pc}$ today.

We computed the light element yields for a variety of initial inhomogeneity profiles and found a region in the parameter space where a low lithium constraint ${ }^{7} \mathrm{Li} / \mathrm{H}<$ $10^{-9.7}$ and a low deuterium constraint $\mathrm{D} / \mathrm{H}<10^{-4.4}$ are satisfied simultaneously for $\eta=(2.1-5.2) \times 10^{-10}$.

\section{ACKNOWLEDGEMENTS}

I thank the Center for Scientific Computing (Finland) for computational resources. 

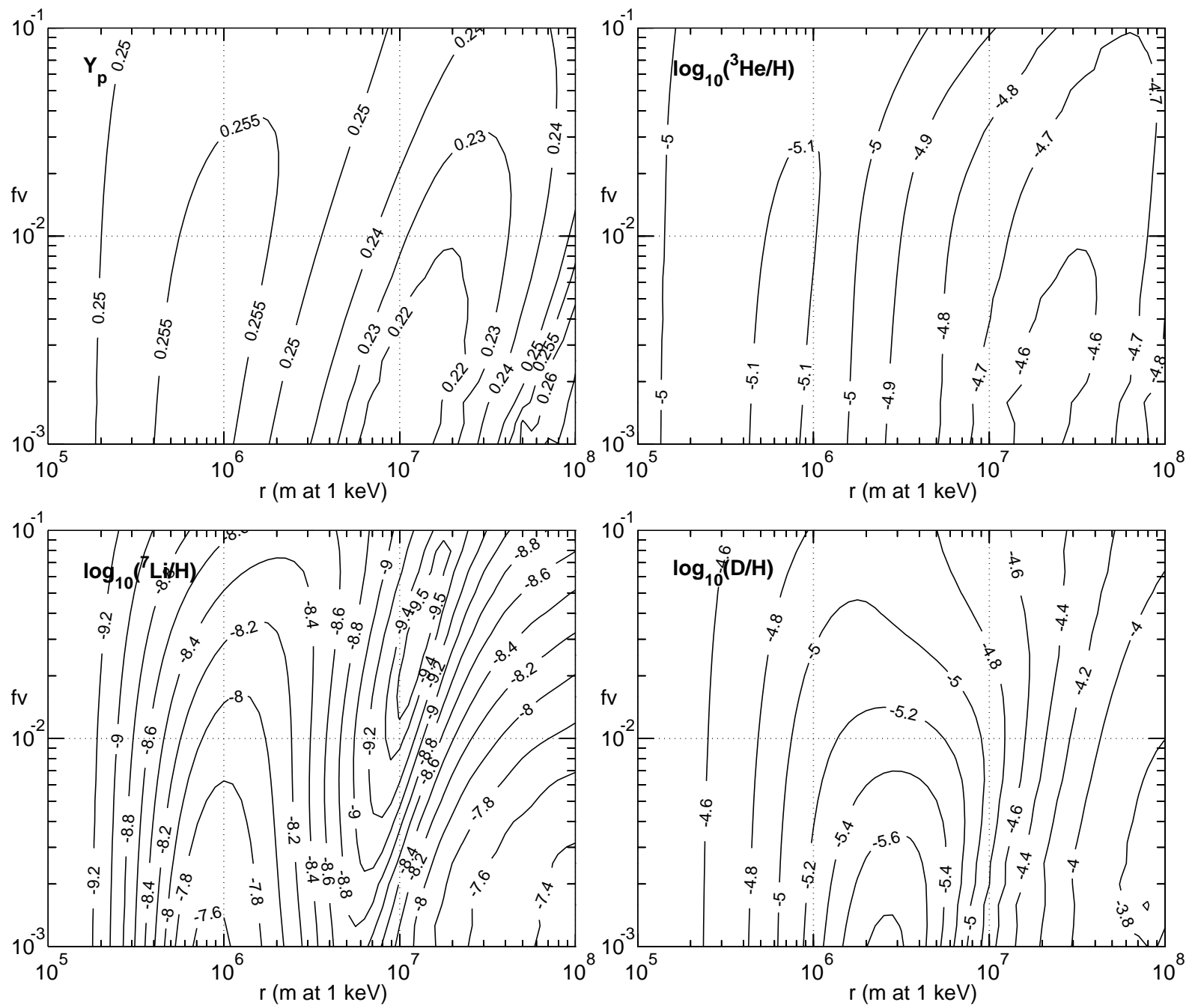

FIG. 4. Isotope yields as a function of the inhomogeneity length scale $r$ and the volume fraction $f_{v}$ of the high-density region. Other simulation parameters were $\eta=6 \times 10^{-10}$ and $R=10^{6}$. At the left the results converge towards SBBN yields $\mathrm{D} / \mathrm{H}=2.9 \times 10^{-5},{ }^{3} \mathrm{He} / \mathrm{H}=1.0 \times 10^{-5}, Y_{p}=0.2483$, and ${ }^{7} \mathrm{Li} / \mathrm{H}=4.1 \times 10^{-10}$. 

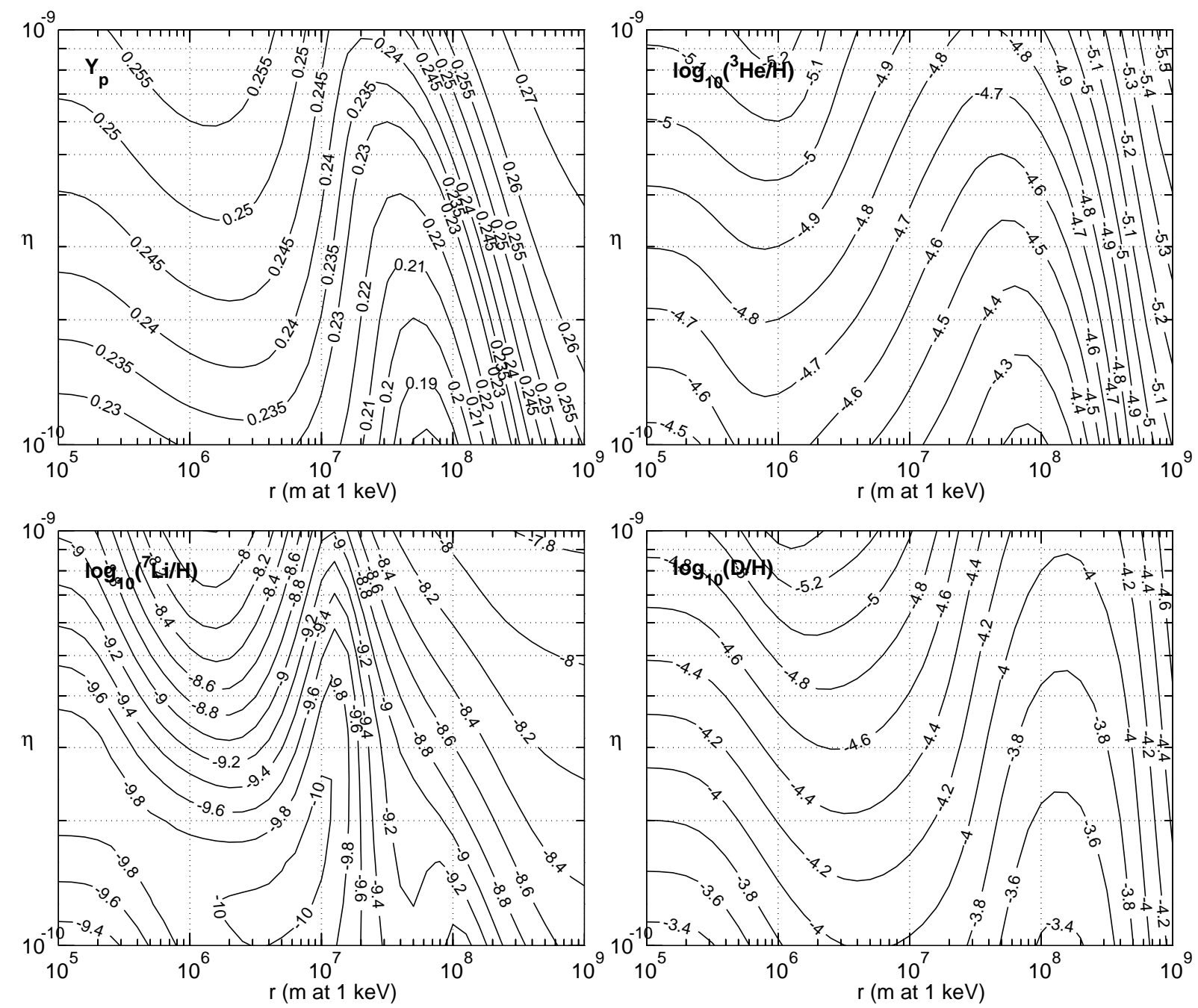

FIG. 5. Isotope yields on $(r, \eta)$ plane for $f_{v}=10^{-1.5}$ and $R=10^{6}$. 
* Electronic address: Elina.Keihanen@helsinki.fi

[1] J.H. Applegate and C.J. Hogan, Phys. Rev. D 31, 3037 (1985).

[2] J.H. Applegate, C.J. Hogan, and R.J. Scherrer, Phys. Rev. D 35, 1151 (1987).

[3] C. Alcock, G.M. Fuller, and G.J. Mathews, Astrophys. J. 320, 439 (1987).

[4] R.M. Malaney and W.A. Fowler, Astrophys. J. 333, 14 (1988).

[5] N. Terasawa and K. Sato, Phys. Rev. D 39, 2893 (1989).

[6] H. Kurki-Suonio, R.A. Matzner, J.M. Centrella, T. Rothman, and J.R. Wilson, Phys. Rev. D 38, 1091 (1988).

[7] H. Kurki-Suonio and R.A. Matzner, Phys. Rev. D 39, 1046 (1989).

[8] H. Kurki-Suonio, R.A. Matzner, K.A. Olive, and D.N. Schramm, Astrophys. J. 353, 406 (1990).

[9] H. Kurki-Suonio and R.A. Matzner, Phys. Rev. D 42, 1047 (1990).

[10] G.J. Mathews, B.S. Meyer, C.R. Alcock, and G.M. Fuller, Astrophys. J. 358, 36 (1990).

[11] G.J. Mathews, D.N. Schramm, and B.S. Meyer, Astrophys. J. 404, 476 (1993).

[12] K. Jedamzik, G.M. Fuller, and G.J. Mathews, Astrophys. J. 423, 50 (1994).

[13] G.J. Mathews, T. Kajino, and M. Orito, Astrophys. J. 456, 98 (1996).

[14] M. Orito, T. Kajino, R.N. Boyd, and G.J. Mathews, Astrophys. J. 488, 515 (1997).

[15] H. Kurki-Suonio, K. Jedamzik, and G.J. Mathews, Astrophys. J. 479, 31 (1997).

[16] K. Kainulainen, H. Kurki-Suonio and E. Sihvola, Phys. Rev. D 59, 083505 (1999).

[17] H. Kurki-Suonio and E. Sihvola, Phys. Rev. D 63, 083508 (2001).

[18] K. Jedamzik and J.B. Rehm, Phys. Rev. D 64, 023510 (2001).

[19] C.R. Alcock, D.S. Dearborn, G.M. Fuller, G.J. Mathews, and B.S. Meyer, Phys. Rev. Lett. 64, 2607 (1990).

[20] K. Jedamzik and G.M. Fuller, Astrophys. J. 423, 33 (1994).

[21] See, e.g., R.D. Present, Kinetic Theory of Gases, (McGraw-Hill Book Company, New York, 1958).

[22] M.A. Preston and R.K. Bhaduri, Structure of the Nucleus (Addison-Wesley, Reading, MA, 1975).

[23] L.D. Landau and E.M. Lifschitz, Physical Kinetics (Pergamon, New York, 1981).

[24] R.J. Goldston and P.H. Rutherford, Introduction to Plasma Physics (IOP, Bristol,1995).

[25] L.D. Landau and E.M. Lifschitz, Fluid Mechanics (Pergamon, New York, 1959).

[26] K.R. Lang, Astrophysical formulae (Springer, Berlin,1980).

[27] P.J.E. Peebles, Physical Cosmology (Princeton University, Princeton, NJ, 1971); P.J.E. Peebles, Principles of Physical Cosmology (Princeton University, Princeton,
NJ, 1993).

[28] L. Spitzer, Jr., Physics of Fully Ionized Gases (Interscience, New York, 1956).

[29] C. Angulo et al. (The NACRE collaboration), Nucl. Phys. A656, 3 (1999).

[30] M. Spite and F. Spite, Astron. Astrophys. 115, 357 (1982); Nature 297, 483 (1982); P. Bonifacio and P. Molaro, Mon. Not. R. Astron. Soc. 285, 847 (1997).

[31] M.H. Pinsonneault, T.P. Walker, G. Steigman, and V.K. Narayanan, Astrophys. J. 527, 180 (1999); M.H. Pinsonneault, G. Steigman, T.P. Walker, and V.K. Narayanan, astro-ph/0105439 (2001).

[32] S.G. Ryan, J.E. Norris, and T.C. Beers, Astrophys. J. 523, 654 (1999).

[33] S.G. Ryan, T.C. Beers, K.A. Olive, B.D. Fields, and J.E. Norris, Astrophys. J. Lett. 530, L57 (2000).

[34] T.K. Suzuki, Y. Yoshii, and T.C. Beers, Astrophys. J. 540, 99 (2000).

[35] P. Bonifacio et al. , astro-ph/0204332 (2002).

[36] J.M. O'Meara, D. Tytler, D. Kirkman, N. Suzuki, J.X. Prochaska, D. Lubin, and A.M. Wolfe, Astrophys. J. 552, 718 (2001).

[37] S. D'Odorico, M. Dessauges-Zavadsky, and P. Molaro, astro-ph/0102162.

[38] M. Pettini and D.V. Bowen, Astrophys. J. 560, 41 (2001).

[39] P. de Bernardis et al. , Astrophys. J. 564, 559 (2002).

[40] J.L. Linsky et al. , Astrophys. J. 402, 694 (1993); J.L. Linsky et al. , Astrophys. J. 451, 335 (1995). 